Paper

\title{
A modified algorithm for accurate inverse Cholesky factorization
}

\author{
Yuka Yanagisawa ${ }^{1 a)}$, Takeshi Ogita ${ }^{2}$, and Shin'ichi Oishi ${ }^{1}$ \\ ${ }^{1}$ Graduate School of Fundamental Science and Engineering, Waseda University \\ 3-4-1 Okubo, Shinjuku-ku, Tokyo 169-8555, Japan \\ ${ }^{2}$ Division of Mathematical Sciences, Tokyo Woman's Christian University \\ 2-6-1 Zempukuji, Suginami-ku, Tokyo 167-8585, Japan \\ a)yuuka@ruri.waseda.jp
}

Received May 20, 2013; Revised August 20, 2013; Published January 1, 2014

\begin{abstract}
This paper is concerned with an inverse matrix factorization based on Cholesky factorization for ill-conditioned matrices. Recently, Ogita and Oishi derived an iterative algorithm to calculate an accurate approximate inverse of the exact Cholesky factor for such matrices. In this paper, a modified version of the algorithm is proposed. It is explained that the proposed algorithm gives more accurate results than the original one by a numerical analysis. Numerical evidence is also shown.
\end{abstract}

Key Words: inverse matrix factorization, Cholesky factorization, ill-conditioned matrix, accurate numerical algorithm

\section{Introduction}

Let $\mathbb{R}$ be the set of real numbers, and $\mathbb{F}$ a set of floating-point numbers conforming IEEE standard 754. The relative rounding error unit of floating-point arithmetic is denoted by $u$. In binary64 (double precision) arithmetic, $u=2^{-53} \approx 1.1 \times 10^{-16}$. Throughout this paper, we assume that neither overflow nor underflow occurs. For $A \in \mathbb{R}^{n \times n}$, define $\kappa(A):=\|A\|\left\|A^{-1}\right\|$ as the condition number of $A$, where $\|\cdot\|$ stands for spectral norm for matrices and Euclidean norm for vectors.

In this paper, we propose an algorithm for an accurate inverse matrix factorization of a given symmetric positive definite matrix $A$ based on Cholesky factorization. We consider to treat the case where $A$ is ill-conditioned such that

$$
\kappa(A)>u^{-1} .
$$

There are several applications in which the case (1) appears, for example, semidefinite programing for optimization problem [1], ill-conditioned Gramian matrices deriving from discretization of partial differential equations and so forth.

In [2], Ogita and Oishi presented an iterative algorithm to calculate a good approximate inverse $X$ of the exact Cholesky factor $\hat{R}$ such that $A=\hat{R}^{T} \hat{R}$ satisfying $\kappa\left(X^{T} A X\right) \approx 1$. Moreover, in $[3$, 4], we presented a detailed analysis showing that if we can use high precision computations for dot product, then the condition number of a preconditioned matrix is reduced by a factor around $n^{2} u$ in each iteration until convergence. 
Cholesky factorization sometimes breaks down by the presence of an imaginary root due to the accumulation of rounding errors, even if the matrix is symmetric and positive definite. To avoid the break-down, a diagonal shift is applied to $A$ in the Ogita-Oishi's algorithm. As we will see from some numerical results in Section 4, the Ogita-Oishi's algorithm gives an approximate inverse $X$ satisfying

$$
n^{2} u \lesssim\left\|I-X^{T} A X\right\|<1 .
$$

The reason why $n^{2} u$ is the lower bound is due to the diagonal shift. To overcome it, we find the condition such that Cholesky factorization of a preconditioned matrix never breaks down without the diagonal shift. In this paper, we propose a modified version of the Ogita-Oishi's algorithm for calculating a more accurate inverse $X$ of $\hat{R}$ than the original one. Namely, the proposed algorithm provides $X$ satisfying

$$
\left\|I-X^{T} A X\right\| \approx u .
$$

The rest of the paper is organized as follows: in the following section, we explain the notation and state the definitions used in this paper. In Section 3, we propose a modified algorithm for an accurate inverse Cholesky factorization. In Section 4, we present some numerical results for comparing the proposed algorithm with the original one in terms of both the accuracy on $X$ and measured computing time for convergence.

\section{Notation and definitions}

Let $I$ denote the identity matrix. For $A=\left(a_{i j}\right), B=\left(b_{i j}\right) \in \mathbb{R}^{n \times n},|A|=\left(\left|a_{i j}\right|\right) \in \mathbb{R}^{n \times n}$ denotes a nonnegative matrix consisting of entrywise absolute values, and an inequality $A \leq B$ is understood entrywise, i.e., $a_{i j} \leq b_{i j}$ for all $(i, j)$. The notation $A \geq O$ means that all elements of $A$ are nonnegative. Similar notation is applied to real vectors. The eigenvalues of $A$ is denoted by $\lambda_{i}(A)$ with $\lambda_{1}(A) \geq \lambda_{2}(A) \geq \cdots \geq \lambda_{n}(A)$. The trace of $A=\left(a_{i j}\right) \in \mathbb{R}^{n \times n}$ is defined by

$$
\operatorname{tr}(A):=\sum_{i=1}^{n} a_{i i}=\sum_{i=1}^{n} \lambda_{i}(A) .
$$

The sum of the absolute values of off-diagonal elements in the $i$-th row of $A$ is denoted by

$$
r_{i}(A)=\sum_{i \neq j}\left|a_{i j}\right| .
$$

The inversion of an upper triangular matrix $R \in \mathbb{F}^{n \times n}$ in solving a matrix equation $R T=I$ for $T$ using a standard numerical algorithm (e.g., xTRSV in BLAS and xTRTRI in LAPACK) is defined by

$$
T:=\operatorname{triinv}(R) .
$$

Let $f(\cdot)$ denote that an expression inside the parentheses is evaluated in floating-point arithmetic. We assume the use of the following standard model: For $a, b \in \mathbb{F}$ and $\circ \in\{+,-, *, /\}$ it holds that

$$
f(a \circ b)=(1+\varepsilon)(a \circ b), \quad|\varepsilon| \leq u .
$$

Let $\mathbb{F}_{\Sigma}$ be a set of sums of floating-point numbers such that

$$
\mathbb{F}_{\Sigma}=\left\{x \in \mathbb{R}: x=\sum_{i=1}^{m} x_{i}, x_{i} \in \mathbb{F}, m \in \mathbb{N}\right\} .
$$

Note that $\mathbb{F} \subseteq \mathbb{F}_{\Sigma} \subset \mathbb{R}$.

For readability we denote by $\varphi(\gamma)$ a constant such as $\varphi(\gamma)=c \cdot \gamma$ where $c:=\mathcal{O}(1)$ with $0<c \ll u^{-1}$.

Let $A, B \in \mathbb{F}_{\Sigma}^{n \times n}$. Assume that we have a function of calculating $C \in \mathbb{F}_{\Sigma}^{n \times n}$ for any $k, l \in \mathbb{N}, l \leq k$ satisfying

$$
|A B-C| \leq \varphi\left(u^{l}\right)|A B|+\varphi\left(u^{k}\right)|A||B| .
$$


Note that $C=\sum_{i=1}^{l} C_{i}$ with $C_{i} \in \mathbb{F}^{n \times n}, i=1,2, \ldots, l$. Namely, $C$ is an approximation of $A B$ as if it is computed in $k$-fold working precision and rounded into $l$ pieces of working precision floating-point numbers. We write such a function as

$$
C_{1: l}=\{A B\}_{k}^{l}
$$

Such accurate dot product algorithms satisfying (3) have been proposed in [7-9]. Moreover, algorithms for accurate matrix multiplication have been developed in [10]. Let $\left\langle A_{M}, A_{R}\right\rangle$ denote an interval matrix of the midpoint-radius representation such that

$$
\left\langle A_{M}, A_{R}\right\rangle:=\left\{X \in \mathbb{R}^{n \times n}:\left|X-A_{M}\right| \leq A_{R}\right\}
$$

with the midpoint $A_{M} \in \mathbb{F}^{n \times n}$ and the radius $A_{R} \in \mathbb{F}^{n \times n}, A_{R} \geq O$.

Similar notation in (4) is applied to $B^{T} A B$, i.e., we assume the availability of a function

$$
\langle G, E\rangle=\left\{B^{T} A B\right\}_{k}^{1}
$$

which satisfies

$$
\left|B^{T} A B-G\right| \leq \varphi(u)\left|B^{T} A B\right|+\varphi\left(u^{k}\right)\left|B^{T}\right||A||B|=E
$$

where $G, E \in \mathbb{F}^{n \times n}$ with $G=G^{T}$ and $E \geq O$.

\section{Modified algorithm for accurate inverse Cholesky factorization}

In this section, we first introduce the Ogita-Oishi algorithm for an accurate inverse Cholesky factorization. After that, we propose a modified version of the Ogita-Oishi's algorithm for calculating a better approximate inverse $X$ of the exact Cholesky factor of $A$ satisfying $\left\|I-X^{T} A X\right\| \approx u$. Moreover, we give a detailed analysis of the proposed algorithm.

\subsection{Preliminaries}

Let $A \in \mathbb{F}^{n \times n}$ be symmetric and positive definite. Throughout the paper, the Matlab-style notation

$$
R=\operatorname{chol}(A)
$$

means a floating-point Cholesky factorization of $A$ using a standard numerical algorithm (e.g., xPOTRF in LAPACK) such that

$$
A \approx R^{T} R
$$

where $R \in \mathbb{F}^{n \times n}$ is an upper triangular matrix. We say "run to completion" if no imaginary root appears in the factorization process. We utilize the backward error analysis of floating-point Cholesky factorization in [12]: if $2(n+1) u<1$, then

$$
\Delta:=A-R^{T} R, \quad\|\Delta\| \leq c_{n}^{\prime} u \cdot \operatorname{tr}(A), \quad c_{n}^{\prime}:=\frac{n+1}{1-2(n+1) u} .
$$

Lemma 1 (cf. Theorem 2.3 in [11]) Let $A=A^{T} \in \mathbb{F}^{n \times n}$. If $\lambda_{n}(A) \geq c_{n}^{\prime} u \cdot \operatorname{tr}(A)$, then $\operatorname{chol}(A)$ runs to completion barring overflow and underflow.

As mentioned before, a floating-point Cholesky factorization of an ill-conditioned matrix $A$ sometimes breaks down due to the accumulation of the rounding errors. To avoid the break-down, a diagonal shift can be applied to $A$ such as $\tilde{A}=f l(A+\delta I)$ for some suitable $\delta>0$. It is advisable to obtain an optimal $\delta$ before executing $\operatorname{chol}(\tilde{A})$.

Assume $A$ is positive definite. Then $a_{i i}>0$ for all $i$ and $\lambda_{n}(A)>0$. From (2), it holds for a diagonal matrix $D=\left(d_{i j}\right) \in \mathbb{R}^{n \times n}$ that

$$
\tilde{A}=A+\delta I+D, \quad\left|d_{i i}\right| \leq u\left(a_{i i}+\delta\right) .
$$


From Lemma 1, if $\lambda_{n}(\tilde{A}) \geq c_{n}^{\prime} u \cdot \operatorname{tr}(\tilde{A})$, then $\operatorname{chol}(\tilde{A})$ never breaks down. Here

$$
\begin{aligned}
c_{n}^{\prime} u \cdot \operatorname{tr}(\tilde{A}) & =c_{n}^{\prime} u \cdot \operatorname{tr}(A+\delta I+D) \leq c_{n}^{\prime} u \cdot \operatorname{tr}(A+\delta I+|D|) \\
& \leq c_{n}^{\prime} u(1+u)(\operatorname{tr}(A)+n \delta) .
\end{aligned}
$$

On the other hand, by a Weyl's theorem (e.g. Corollary 4.9 in [13]) it holds

$$
\lambda_{n}(\tilde{A}) \geq \lambda_{n}(A+\delta I)-\|D\|>\delta-\max _{1 \leq i \leq n}\left|d_{i i}\right|,
$$

and

$$
\max _{1 \leq i \leq n}\left|d_{i i}\right| \leq \max _{1 \leq i \leq n} u\left(a_{i i}+\delta\right)=u \max _{1 \leq i \leq n} a_{i i}+\delta u \leq u \cdot \operatorname{tr}(A)+\delta u
$$

so that

$$
\lambda_{n}(\tilde{A}) \geq \delta-(u \cdot \operatorname{tr}(A)+\delta u)=(1-u) \delta-u \cdot \operatorname{tr}(A) .
$$

From (7) and (8), a sufficient condition such that $\operatorname{chol}(\tilde{A})$ runs to completion is

$$
(1-u) \delta-u \cdot \operatorname{tr}(A) \geq c_{n}^{\prime} u(1+u)(\operatorname{tr}(A)+n \delta)
$$

and

$$
\delta \geq \frac{(1+u) c_{n}^{\prime}+1}{1-\left((1+u) n c_{n}^{\prime}+1\right) u} u \cdot \operatorname{tr}(A) .
$$

If $(n+1)(n+3) u<1$, then a little computation yields

$$
\frac{(1+u) c_{n}^{\prime}+1}{1-\left((1+u) n c_{n}^{\prime}+1\right) u} \leq \frac{n+2}{1-(n+1)(n+3) u}=: c_{n} .
$$

Thus, if we determine $\delta$ satisfying $\delta \geq c_{n} u \cdot \operatorname{tr}(A)$, then $\operatorname{chol}(\tilde{A})$ runs to completion.

If $(n+1)(n+3) u<1$, then we define a diagonal $\operatorname{shift}^{1}$ of $A \in \mathbb{F}^{n \times n}$ by

$$
\operatorname{shift}(A):=c_{n} u \cdot \operatorname{tr}(A) \quad \text { with } \quad c_{n}=\frac{n+2}{1-(n+1)(n+3) u} .
$$

Theorem 1 Let $A=A^{T} \in \mathbb{F}^{n \times n}$. Let $\operatorname{shift}(A)$ be defined as in (10). For any positive $\delta \in \mathbb{F}$ satisfying $\delta \geq \operatorname{shift}(A)$, put $\tilde{A}:=f l(A+\delta I)$. If $A$ is positive definite, then $\operatorname{chol}(\tilde{A})$ runs to completion barring overflow and underflow.

The following is the original Ogita-Oishi's algorithm:

Algorithm 1 (Ogita-Oishi [2]) For a symmetric matrix $A=\left(a_{i j}\right) \in \mathbb{F}_{\Sigma}^{n \times n}$ with $a_{i i}>0$ for all $i$ and a specified tolerance $\varepsilon_{t o l}$ satisfying $n^{2} u \lesssim \varepsilon_{t o l} \leq 1$, the following algorithm calculates an upper triangular matrix $X_{1: m_{k}}^{(k)} \in \mathbb{F}_{\Sigma}^{n \times n}$ for some $k \in \mathbb{N}$ such that $\left\|X_{1: m_{k}}^{(k) T} A X_{1: m_{k}}^{(k)}-I\right\|<\varepsilon_{\text {tol }}$.

$$
\begin{aligned}
& k=0, \quad G^{(0)}:=A_{1: 1}, \quad E^{(0)}:=O, \quad X_{1: 1}^{(0)}:=I \\
& \text { repeat } \\
& \quad k=k+1 \\
& \quad \delta_{k}:=\operatorname{shift}\left(G^{(k-1)}\right)+\left\|E^{(k-1)}\right\| \\
& \quad \text { Compute } S^{(k)} \in \mathbb{F}^{n \times n} \text { with } \quad S_{i i}^{(k)} \geq G_{i i}^{(k-1)}+\delta_{k}, \quad S_{i j}^{(k)}=G_{i j}^{(k-1)} \text { for } i \neq j . \\
& \quad R^{(k)}:=\operatorname{chol}\left(f\left(S^{(k)}+\delta_{k} I\right)\right) \\
& \quad T^{(k)}:=\operatorname{triinv}\left(R^{(k)}\right) \\
& \quad X_{1: m_{k}}^{(k)}:=\left\{X_{1: m_{k-1}}^{(k-1)} T^{(k)}\right\}_{m_{k}}^{m_{k}} \quad \% m_{k}:=\left\lceil\frac{k}{2}\right\rceil+1 \\
& \quad\left\langle G^{(k)}, E^{(k)}\right\rangle:=\left\{X_{1: m_{k}}^{(k) T} A X_{1: m_{k}}^{(k)}\right\}_{k+1}^{1} \\
& \text { until } \quad\left\|G^{(k)}-I\right\|+\left\|E^{(k)}\right\|<\varepsilon_{t o l}
\end{aligned}
$$

\footnotetext{
${ }^{1}$ In $[2,3]$, we applied a similar diagonal shift, which is modified for the sake of completeness in this paper.
} 
Remark 1 To ensure the positive definiteness of $G^{(k-1)}$ with taking care of the rounding errors, we slightly shift the diagonal part of $G^{(k-1)}$ by $\delta_{k}$ in Algorithm 1 . If it holds that $\left\|E^{(k-1)}\right\| \approx u\left\|G^{(k-1)}\right\|$, then

$$
\begin{aligned}
\delta_{k} & =c_{n} u \cdot \operatorname{tr}\left(G^{(k-1)}\right)+\left\|E^{(k-1)}\right\| \approx n u \cdot \operatorname{tr}\left(G^{(k-1)}\right)+u\left\|G^{(k-1)}\right\| \\
& \lesssim n^{2} u\left\|G^{(k-1)}\right\| .
\end{aligned}
$$

If that is the case, then we have

$$
\alpha:=\frac{\delta_{k}}{\left\|G^{(k-1)}\right\|} \lesssim n^{2} u
$$

Remark 2 For (12), in practice, $S^{(k)}$ can be obtained by calculating $G_{i i}^{(k-1)}+\delta_{k}$ in rounding upwards.

Remark 3 Since $S^{(k)} \approx G^{(k-1)}+\delta_{k} I$, we use $G^{(k-1)}+\delta_{k} I$ instead of $S^{(k)}$ in the following.

Remark 4 From Analysis 2 in [3], we can see that the condition number of a preconditioned matrix is reduced by a factor around $\alpha$ in each iteration as follows:

$$
\kappa\left(X_{1: m_{k}}^{(k) T} A X_{1: m_{k}}^{(k)}\right) \approx \alpha^{k} \kappa(A)
$$

Remark 5 From (16), we can see that the number of iterations $m_{1}$ in Algorithm 1 is estimated as

$$
m_{1}=\left\lceil\frac{\log \varepsilon_{t o l}-\log \kappa(A)}{\log \alpha}\right\rceil
$$

We consider the accuracy on $X_{1: m_{k}}^{(k)}$ provided by Algorithm 1. For readability, $X_{1: m_{k}}^{(k)}$ is abbreviated to $X$. Define $c_{\alpha}:=\min \left(\alpha^{-1}, \kappa\left(X^{T} A X\right)\right)$. From Analysis 2 in [3], it holds that

$$
\left\|I-X^{T} A X\right\| \approx \alpha c_{\alpha}
$$

If $\kappa\left(X^{T} A X\right) \geq \alpha^{-1}$, then the definition of $c_{\alpha}$ implies $c_{\alpha}=\alpha^{-1}$ and $\left\|I-X^{T} A X\right\| \approx 1$. Otherwise, $c_{\alpha}=\kappa\left(X^{T} A X\right)$ and $\left\|I-X^{T} A X\right\| \lesssim 1$. Moreover, in the best case as $\kappa\left(X^{T} A X\right)=1$ we have $c_{\alpha}=1$ and $\left\|I-X^{T} A X\right\| \approx \alpha$. Therefore, it holds that

$$
\alpha \lesssim\left\|I-X^{T} A X\right\| \lesssim 1
$$

The reason why $\alpha$ is the lower bound is due to the diagonal shift (11). On (15), from Analysis 2 in [3], we have $\left\|G^{(k-1)}\right\| \approx 1$. Then it holds that $\delta_{k} \approx \alpha$.

\subsection{Proposed algorithm}

The following is a modified algorithm for an accurate inverse Cholesky factorization:

Algorithm 2 (mAccInvChol) For a symmetric matrix $A=\left(a_{i j}\right) \in \mathbb{F}_{\Sigma}^{n \times n}$ with $a_{i i}>0$ for all $i$, the following algorithm calculates an upper triangular matrix $X_{1: m_{M}}^{(M)} \in \mathbb{F}_{\Sigma}^{n \times n}$ where $M:=k+1$ for some $k \in \mathbb{N}$ such that $\left\|X_{1: m_{M}}^{(M) T} A X_{1: m_{M}}^{(M)}-I\right\| \approx u$. 


$$
\begin{aligned}
& k=0, \quad G^{(0)}:=A_{1: 1}, \quad E^{(0)}:=O, \quad X_{1: 1}^{(0)}:=I \\
& \text { repeat } \\
& k=k+1 \\
& \delta_{k}:=\operatorname{shift}\left(G^{(k-1)}\right)+\left\|E^{(k-1)}\right\| \\
& \text { Compute } S^{(k)} \in \mathbb{F}^{n \times n} \text { with } S_{i i}^{(k)} \geq G_{i i}^{(k-1)}+\delta_{k}, \quad S_{i j}^{(k)}=G_{i j}^{(k-1)} \text { for } i \neq j \text {. } \\
& R^{(k)}:=\operatorname{chol}\left(f l\left(S^{(k)}+\delta_{k} I\right)\right) \\
& T^{(k)}:=\operatorname{triinv}\left(R^{(k)}\right) \\
& X_{1: m_{k}}^{(k)}:=\left\{X_{1: m_{k-1}}^{(k-1)} T^{(k)}\right\}_{m_{k}}^{m_{k}} \quad \% m_{k}:=\left\lceil\frac{k}{2}\right\rceil+1 \\
& \left\langle G^{(k)}, E^{(k)}\right\rangle:=\left\{X_{1: m_{k}}^{(k) T} A X_{1: m_{k}}^{(k)}\right\}_{k+1}^{1} \\
& \beta:=\min \left(G_{i i}^{(k)}-r_{i}\left(G^{(k)}\right)\right) \\
& \mu:=c_{n}^{\prime} u \cdot \operatorname{tr}\left(G^{(k)}\right) \quad \% c_{n}^{\prime}:=\frac{n+1}{1-2(n+1) u} \\
& \text { until } \beta>\max \left(\mu,\left\|E^{(k)}\right\|\right) \\
& R^{(M)}:=\operatorname{chol}\left(G^{(M-1)}\right) \quad \% M:=k+1 \\
& T^{(M)}:=\operatorname{triinv}\left(R^{(M)}\right) \\
& X_{1: m_{M}}^{(M)}:=\left\{X_{1: m_{k}}^{(M-1)} T^{(M)}\right\}_{m_{M}}^{m_{M}} \quad \% m_{M}:=\left\lceil\frac{k+1}{2}\right\rceil+1
\end{aligned}
$$

Remark 6 We can replace (19) with

$$
R^{(k)}=\operatorname{chol}\left(G^{(k-1)}\right)
$$

which means the diagonal shift is not applied. If (22) breaks down, then we adopt (19). Then it is expected that Algorithm 2 converges faster.

Remark 7 Algorihtm 2 can be applied to interval matrices if accurate matrix multiplication (20) accepts interval inputs.

Compared with Algorithm 1, we add the condition such that Cholesky factorization of $G^{(k-1)}$ never breaks down without a diagonal shift (11). In the following, we explain how to determine it. For readability, $G^{(k)}, X_{1: m_{k}}^{(k)}$ and $X_{1: m_{M}}^{(M)}$ where $M:=k+1$ are abbreviated to $G, X$ and $X^{\prime}$, respectively. Let $R^{\prime}$ be a computed Cholesky factor of $G$, i.e., $R^{\prime}=\operatorname{chol}(G)$. Define

$$
\begin{aligned}
& \Delta_{1}:=G-X^{T} A X, \\
& \Delta_{2}:=G-R^{\prime T} R^{\prime}, \\
& T:=\operatorname{triinv}\left(R^{\prime}\right), \\
& \Delta_{3}:=X^{\prime}-X T, \\
& c_{\alpha}:=\min \left(\alpha^{-1}, \kappa\left(X^{T} A X\right)\right) .
\end{aligned}
$$

By the definition (24), it is necessary to satisfy

$$
\lambda_{n}\left(G-\Delta_{2}\right)>0 .
$$

By (6) and a Weyl's theorem (e.g. Corollary 4.9 in [13]) for (24), it holds that

$$
\lambda_{n}\left(G-\Delta_{2}\right) \geq \lambda_{n}(G)-\left\|\Delta_{2}\right\|, \quad\left\|\Delta_{2}\right\| \leq c_{n}^{\prime} u \cdot \operatorname{tr}(G) .
$$

The Gerschgorin's circle theorem implies

$$
\lambda_{k}(G) \in \bigcup_{i=1}^{n}\left\{x \in \mathbb{R}:\left|x-G_{i i}\right| \leq r_{i}(G)\right\}, \quad k=1,2, \ldots, n
$$


and

$$
\lambda_{n}(G) \geq \beta \quad \text { where } \quad \beta:=\min \left(G_{i i}-r_{i}(G)\right) .
$$

From (28) and (29), we have

$$
\lambda_{n}\left(G-\Delta_{2}\right) \geq \beta-\left\|\Delta_{2}\right\| .
$$

Moreover, to ensure the positive definiteness of $A$ with taking care of the rounding errors, it is necessary to satisfy

$$
\lambda_{n}\left(X^{T} A X\right)>0
$$

By a Weyl's theorem (e.g. Corollary 4.9 in [13]) for (23), it holds that

$$
\lambda_{n}\left(X^{T} A X\right) \geq \lambda_{n}(G)-\left\|\Delta_{1}\right\| .
$$

From (29), we have

$$
\lambda_{n}\left(X^{T} A X\right) \geq \beta-\left\|\Delta_{1}\right\| .
$$

Therefore, combining (30) and (31), the sufficient condition to make $\operatorname{chol}(G)$ run to completion and to ensure the positive definiteness of $A$ is

$$
\beta>\max \left(\left\|\Delta_{1}\right\|,\left\|\Delta_{2}\right\|\right) .
$$

\subsection{Analysis of the accuracy on $X$}

The target of this subsection is to explain that $\left\|X^{(M) T} A X^{(M)}-I\right\| \approx u$ if $G^{(k)}$ satisfy (32) at the $k$-th iteration. For later use, we present the following observation.

Observation 1 Let $A=\left(a_{i j}\right) \in \mathbb{F}^{n \times n}$ be symmetric and positive definite. Define $A=I+E$. Let $E_{U}$ be the strictly upper triangular part of $E$. Suppose $\|E\| \leq \alpha \ll 1$. Let a triangular matrix $R=\left(r_{i j}\right) \in \mathbb{F}^{n \times n}$ be a computed Cholesky factor of $A$, i.e., $R=\operatorname{chol}(A)$. Then

$$
R \approx I+E_{U}, \quad\left\|E_{U}\right\| \leq \alpha \quad \text { and } \quad\left\|A-R^{T} R\right\| \lesssim u+n \alpha^{2} .
$$

Argument First, we estimate diagonal and off-diagonal elements of $R$, respectively. Here $\mathrm{fl}(\cdot)$ means that arithmetic inside the parenthesis is done in binary64 floating point arithmetic. It follows that

$$
\begin{aligned}
& r_{j j}=\mathrm{fl}\left(\left(a_{j j}-\sum_{k=1}^{j-1} r_{k j}^{2}\right)^{\frac{1}{2}}\right)=(1+\epsilon)\left(a_{j j}-\epsilon_{1}\right)^{\frac{1}{2}}=(1+\epsilon)\left(1+\epsilon^{\prime}\right)^{\frac{1}{2}} \approx 1+\frac{1}{2} \epsilon^{\prime} \\
& |\epsilon| \leq u, \quad\left|\epsilon_{1}\right| \lesssim(n-1) \alpha^{2}, \quad\left|\epsilon^{\prime}\right| \lesssim \alpha,
\end{aligned}
$$

and

$$
r_{i j}=\mathrm{fl}\left(\left(a_{i j}-\sum_{k=1}^{i-1} r_{k i} r_{k j}\right) / r_{i i}\right)=a_{i j}+\epsilon_{2} \quad \text { for } \quad i<j, \quad\left|\epsilon_{2}\right| \lesssim \alpha^{2} .
$$

Thus, we have $r_{j j} \lesssim 1+\frac{1}{2} \alpha$ and $\left|r_{i j}\right| \lesssim \alpha$. Then $R \approx I+E_{U}$.

Next, we estimate $\left\|A-R^{T} R\right\|$. On the off-diagonal elements, it holds [5, p.197] that

$$
\left|a_{i j}-\sum_{k=1}^{i} r_{k i} r_{k j}\right| \leq \gamma_{i} \sum_{k=1}^{i}\left|r_{k i}\right|\left|r_{k j}\right| \quad \text { where } \quad \gamma_{i}=\frac{i u}{1-i u} .
$$

Here, from (33) and (34), we have 


$$
\begin{aligned}
& \left|a_{i j}-\sum_{k=1}^{i} r_{k i} r_{k j}\right| \leq \gamma_{i}\left(\sum_{k=1}^{i-1}\left|r_{k i}\right|\left|r_{k j}\right|+\left|r_{i i}\right|\left|r_{i j}\right|\right) \\
& \lesssim(n-1) u\left((n-2) \alpha^{2}+\left(1+\frac{1}{2} \alpha\right) \alpha\right) \approx n u \alpha .
\end{aligned}
$$

From (33), $r_{j j}^{2}=(1+\epsilon)^{2}\left(a_{j j}-\epsilon_{1}\right) \approx(1+2 \epsilon) a_{j j}$. Hence, it holds that

$$
\begin{aligned}
\left|a_{j j}-\sum_{k=1}^{j} r_{k j}^{2}\right| & \leq\left|a_{j j}-r_{j j}^{2}\right|+\sum_{k=1}^{j-1} r_{k j}^{2} \lesssim\left|a_{j j}-(1+2 \epsilon) a_{j j}\right|+(n-1) \alpha^{2} \\
& \approx 2|\epsilon| a_{j j}+n \alpha^{2} \approx u+n \alpha^{2} .
\end{aligned}
$$

Therefore, we have $\left\|A-R^{T} R\right\| \lesssim n u \alpha+u+n \alpha^{2} \approx u+n \alpha^{2}$. If $n \alpha^{2} \lesssim u$, then $\left\|A-R^{T} R\right\| \lesssim u$.

Observation 2 Let $R$ and $E_{U}$ be defined as in Observation 1. Let $T:=\operatorname{triinv}(R)$. Then

$$
T \approx I-E_{U} \text { and }\|T\| \approx 1 .
$$

Argument From Observation 1, it follows that $R \approx I+E_{U}$ and $\left\|E_{U}\right\| \ll 1$. Then

$$
T=\left(I+E_{U}\right)^{-1}=I-E_{U}+E_{U}^{2}-E_{U}^{3}+\cdots \approx I-E_{U}
$$

and $\|T\| \approx 1$.

Observation 3 Let $R, T$ and $E_{U}$ be defined as in Observations 1 and 2. Then

$$
\left\|I-T^{T} R^{T} R T\right\| \lesssim \alpha^{2} .
$$

Argument From Observations 1 and 2, it follows that

$$
R T \approx\left(I+E_{U}\right)\left(I-E_{U}\right)=I-E_{U}^{2}
$$

and

$$
\left\|I-T^{T} R^{T} R T\right\| \approx\left\|I-\left(I-E_{U}^{2}\right)^{T}\left(I-E_{U}^{2}\right)\right\| \approx 2\left\|E_{U}^{2}\right\| \leq 2 \alpha^{2} .
$$

We estimate $\left\|I-X^{\prime T} A X^{\prime}\right\|$. To explain it, we utilize (23)-(27) in Subsection 3.2. Assume $X$ is nearly the best case as $\kappa\left(X^{T} A X\right) \approx 1$. Then the definition of $c_{\alpha}$ implies $c_{\alpha} \approx 1$. From Analysis 2 in [3], we have $\|G\| \approx 1,\|X\| \approx\left(\alpha^{-k} /\|A\|\right)^{\frac{1}{2}}$ and $\left\|X^{-1}\right\| \approx\|A\|^{\frac{1}{2}}$. The computation of $X^{\prime}$ in $m_{M^{-} \text {-fold }}$ working precision gives

$$
\left\|\Delta_{3}\right\| \leq u^{\left\lceil\frac{k+1}{2}\right\rceil+1}\|X\|\|T\| \approx u^{\left\lceil\frac{k+1}{2}\right\rceil+1}\left(\frac{\alpha^{-k}}{\|A\|}\right)^{\frac{1}{2}} .
$$

Then

$$
\begin{aligned}
\left\|I-X^{\prime T} A X^{\prime}\right\| & =\left\|I-\left(X T+\Delta_{3}\right)^{T} A\left(X T+\Delta_{3}\right)\right\| \\
& \leq\left\|I-T^{T} X^{T} A X T\right\|+2\left\|T^{T} X^{T} A \Delta_{3}\right\|+\left\|\Delta_{3}^{T} A \Delta_{3}\right\| .
\end{aligned}
$$

Here

$$
\begin{aligned}
\left\|I-T^{T} X^{T} A X T\right\| & =\left\|\left(I-T^{T} G T\right)-T^{T} \Delta_{1} T\right\| \\
& =\left\|\left(I-T^{T}\left(R^{\prime T} R^{\prime}+\Delta_{2}\right) T\right)-T^{T} \Delta_{1} T\right\| \\
& \leq\left\|I-T^{T} R^{\prime T} R^{\prime} T\right\|+\left\|T^{T} \Delta_{2} T\right\|+\left\|T^{T} \Delta_{1} T\right\| .
\end{aligned}
$$

From Observation 3, we have 


$$
\left\|I-T^{T} R^{\prime T} R^{\prime} T\right\| \lesssim \alpha^{2} .
$$

Since (5) is used for calculating $X^{T} A X$, it holds that

$$
\left\|\Delta_{1}\right\| \approx u\|G\| \approx u .
$$

From Observation 1, we have

$$
\left\|\Delta_{2}\right\| \approx u+n \alpha^{2} .
$$

From Observation 2, (39) and (40), it follows that

$$
\left\|T^{T} \Delta_{2} T\right\| \leq\|T\|^{2}\left\|\Delta_{2}\right\| \approx u+n \alpha^{2}, \quad\left\|T^{T} \Delta_{1} T\right\| \leq\|T\|^{2}\left\|\Delta_{1}\right\| \approx u .
$$

Inserting (38) and (41) into (37) yields

$$
\left\|I-T^{T} X^{T} A X T\right\| \approx u+n \alpha^{2} .
$$

Moreover, we estimate $\left\|T^{T} X^{T} A \Delta_{3}\right\|$ and $\left\|\Delta_{3}^{T} A \Delta_{3}\right\|$ in (36). By Observation 2, (35) and (39), it holds

$$
\begin{aligned}
\left\|T^{T} X^{T} A \Delta_{3}\right\| & =\left\|T^{T} X^{T} A X X^{-1} \Delta_{3}\right\|=\left\|T^{T}\left(G-\Delta_{1}\right) X^{-1} \Delta_{3}\right\| \\
& \leq\|T\|\left(\|G\|+\left\|\Delta_{1}\right\|\right)\left\|X^{-1}\right\|\left\|\Delta_{3}\right\| \approx u^{\left\lceil\frac{k+1}{2}\right\rceil+1} \alpha^{-\frac{k}{2}} .
\end{aligned}
$$

and

$$
\left\|\Delta_{3}^{T} A \Delta_{3}\right\| \leq\left\|\Delta_{3}\right\|^{2}\|A\| \approx u^{k+3} \alpha^{-k} .
$$

Inserting (42)-(44) into (36) implies

$$
\left\|I-X^{\prime T} A X^{\prime}\right\| \lesssim u+n \alpha^{2}+u^{\left\lceil\frac{k+1}{2}\right\rceil+1} \alpha^{-\frac{k}{2}}+u^{k+3} \alpha^{-k} \approx u+n \alpha^{2} .
$$

If $n^{5} u<1$ satisfies, then $n \alpha^{2} \lesssim u$ and

$$
\left\|I-X^{\prime T} A X^{\prime}\right\| \approx u
$$

Here, $n \alpha^{2}$ in (45) is an upper bound of the rounding errors. In practice, $n \alpha^{2} \approx u$ for larger $n$.

\subsection{Computational complexity}

We assume accurate dot product based on the algorithm in [7-9] is used in Algorithm 2. It requires $\mathcal{O}\left(k^{2} n^{3}\right)$ flops to compute the matrix multiplication $X^{(k-1)} T^{(k)}$ and $X^{(k) T} A X^{(k)}$ and $\mathcal{O}\left(n^{3}\right)$ flops to compute the rest of the algorithm at the $k$-th iteration. If the iterations stop at $k=m_{2}=M-1$, then the computational cost of the part of Algorithm 2 becomes $\mathcal{O}\left(m_{2}^{3} n^{3}\right)$ flops since $\sum_{k=1}^{m_{2}} k^{2} n^{3}=$ $\mathcal{O}\left(m_{2}^{3} n^{3}\right)$. After the iterations, it requires $\mathcal{O}\left(M^{2} n^{3}\right)$ flops to compute the matrix multiplication $X^{(M-1)} T^{(M)}$ and $\mathcal{O}\left(n^{3}\right)$ flops to compute the rest of the algorithm. Then, the total cost of Algorithm 2 becomes $\mathcal{O}\left(M^{3} n^{3}\right)$ flops. From (16), we can see that the number of iterations $m_{2}$ in Algorithm 2 is estimated as

$$
m_{2}=\left\lceil\frac{\log \kappa(A)}{\log \alpha}\right\rceil, \quad \text { and } \quad M=m_{2}+1
$$

We will confirm it in Section 4.

\section{Numerical experiments}

In this section we present some numerical results. All computations are done on Matlab 2012b using IEEE standard 754 binary64 (double precision) on Mac OS X version 10.8 with $2 \mathrm{GHz}$ Intel Core i7 Duo processor, so that $u=2^{-53} \approx 1.11 \times 10^{-16}$. 
Table I. Results for scaled Hilbert matrix $\kappa(A) \approx 8.16 \cdot 10^{29}, \alpha \approx 4.9 \cdot 10^{-14}$ and $\varepsilon_{\text {tol }}=10^{-13}$.

\begin{tabular}{cccccc}
\hline & & & Algorithm 1 & & Algorithm 2 \\
$\kappa$ & $\kappa\left(G^{(k)}\right)$ & $\alpha^{k} \kappa(A)$ & $\left\|I-G^{(k)}\right\|$ & & $\left\|I-G^{(k)}\right\|$ \\
\hline 1 & $2.61 \cdot 10^{15}$ & $4.19 \cdot 10^{16}$ & $1.00 \cdot 10^{00}$ & & $1.00 \cdot 10^{00}$ \\
2 & $8.35 \cdot 10^{01}$ & $2.15 \cdot 10^{03}$ & $9.88 \cdot 10^{-01}$ & & $9.88 \cdot 10^{-01}$ \\
3 & $1.00 \cdot 10^{00}$ & $1.10 \cdot 10^{-10}$ & $4.04 \cdot 10^{-12}$ & & $4.04 \cdot 10^{-12}$ \\
4 & $1.00 \cdot 10^{00}$ & $5.65 \cdot 10^{-24}$ & $5.13 \cdot 10^{-14}$ & & $3.79 \cdot 10^{-16}$ \\
\hline
\end{tabular}

Table II. Result for Rump matrix with $n=500, \kappa(A) \approx 4.756 \times 10^{53}$, $\alpha \approx 2.78 \cdot 10^{-11}$ and $\varepsilon_{t o l}=10^{-10}$.

\begin{tabular}{cccccc}
\hline & & & Algorithm 1 & & Algorithm 2 \\
\cline { 6 - 6 } \cline { 5 - 6 }$\kappa$ & $\kappa\left(G^{(k)}\right)$ & $\alpha^{k} \kappa(A)$ & $\left\|I-G^{(k)}\right\|$ & & $\left\|I-G^{(k)}\right\|$ \\
\hline 1 & $5.95 \cdot 10^{41}$ & $1.32 \cdot 10^{43}$ & $1.00 \cdot 10^{00}$ & & $1.00 \cdot 10^{00}$ \\
2 & $1.38 \cdot 10^{31}$ & $3.68 \cdot 10^{32}$ & $1.00 \cdot 10^{00}$ & & $1.00 \cdot 10^{00}$ \\
3 & $3.59 \cdot 10^{20}$ & $1.02 \cdot 10^{22}$ & $1.00 \cdot 10^{00}$ & & $1.00 \cdot 10^{00}$ \\
4 & $9.71 \cdot 10^{09}$ & $2.85 \cdot 10^{11}$ & $1.00 \cdot 10^{00}$ & & $1.00 \cdot 10^{00}$ \\
5 & $1.27 \cdot 10^{00}$ & $7.91 \cdot 10^{00}$ & $2.11 \cdot 10^{-01}$ & & $2.11 \cdot 10^{-01}$ \\
6 & $1.00 \cdot 10^{00}$ & $2.20 \cdot 10^{-10}$ & $3.52 \cdot 10^{-11}$ & & $3.88 \cdot 10^{-16}$ \\
\hline
\end{tabular}

We present some numerical results for comparing the proposed algorithm (Algorithm 2) with the original one (Algorithm 1), in terms of both the accuracy on $X$ and measured computing time. For Algorithms 1 and 2, we adopt accurate matrix multiplication algorithms in [10]. Then the computations are automatically parallelized in BLAS and LAPACK. Put $G^{(k)}$ from (13) and (20).

First, we observe some numerical results in terms of the accuracy on $X$. From (18), the lower bound of $\left\|I-X^{T} A X\right\|$ in Algorithm 1 is about $n^{2} u$. Thus, we set $\varepsilon_{t o l}=10^{\left\lceil\log _{10} n^{2} u\right\rceil}$ as a stopping criterion for Algorithm 1. We deal with the Hilbert matrix as the first example. Due to rounding errors, the condition number of a floating-point Hilbert matrix is limited not far from $u^{-1}$. Therefore we use the Hilbert matrix scaled by the least common multiple of the denominators as follows:

$$
H_{n}=\left(h_{i j}\right) \in \mathbb{F}^{n \times n} \quad \text { with } \quad h_{i j}:=\operatorname{lcm}(1,2, \cdots, 2 n-1) /(i+j-1) .
$$

Here $H_{n}$ is an integer matrix whose elements are exactly representable in binary64 floating-point numbers for $n \leq 21$ with $\kappa\left(H_{21}\right)=8.16 \cdot 10^{29}$. The results of Algorithm 1 for $A=H_{21}$ are shown in Table I. As the second example, we deal with the Rump matrix [14] as an ill-conditioned matrix, which is based on the function randmat in INTLAB [15], and surely generates symmetric and positive definite matrices. We name the function randmatsym $\left(n\right.$, cnd). We set $n=500$ with cnd $=10^{50}$. Then $A \in \mathbb{F}^{500 \times 500}$ with $\kappa(A) \approx 4.756 \times 10^{53}$ is generated, and the results are shown in Table II. Moreover, we set $n=1000$ with $c n d=10^{100}$. Then $A \in \mathbb{F}^{1000 \times 1000}$ with $\kappa(A) \approx 8.297 \times 10^{102}$ is generated, and the results are shown in Table III.

Let $m_{1}$ and $m_{2}$ denote the number of iterations for Algorithm 1 and that for Algorithm 2, respectively. Put $M=m_{2}+1$. On Algorithm 1, it can be seen from Tables I - III that $\kappa\left(G^{(k)}\right)$ is reduced by a factor around $\alpha$ in each iteration until $\left\|I-G^{\left(m_{1}\right)}\right\| \approx \alpha$, which is consistent with (16) and (18) as expected. Moreover, we can observe that $\left\|I-G^{\left(m_{1}\right)}\right\|$ in Algorithm 1 increases according to the dimension $n$, while $\left\|I-G^{(M)}\right\|$ from Algorithm 2 in Tables I - III is always around $u$, which is consistent with (46) as expected. Thus, Algorithm 2 gives more accurate results than Algorithm 1. As can be seen, in Table III, Algorithm 2 requires less iterations than Algorithm 1. The reason is that the stopping criterion (21) in Algorithm 2 is satisfied when $\kappa\left(G^{\left(m_{2}\right)}\right) \approx 1$ and the iterations finish, while Algorithm 1 continues one more iteration until satisfying $\left\|I-G^{(k)}\right\| \approx \alpha$ even if $\kappa\left(G^{(k)}\right) \approx 1$.

Next, we observe the measured computing time for the algorithms. We again use randmatsym $(n, c n d)$. Here, $t_{1}$ and $t_{2}$ denote the computing time for Algorithm 1 and that for Algorithm 2, respectively. For reference, let $t_{0}$ and $m_{0}$ denote the computing time and the number of iterations for Algorithm 1 with setting $\varepsilon_{t o l}=10^{-6}$, respectively. First, we fix $n=500$ and vary anticipated 
Table III. Result for Rump matrix with $n=1000, \kappa(A) \approx 8.297 \times 10^{102}$, $\alpha \approx 1.11 \cdot 10^{-10}$ and $\varepsilon_{\text {tol }}=10^{-9}$.

\begin{tabular}{cccccc}
\hline & & & Algorithm 1 & & Algorithm 2 \\
\cline { 6 - 6 } \cline { 5 - 6 }$\kappa$ & $\kappa\left(G^{(k)}\right)$ & $\alpha^{k} \kappa(A)$ & $\left\|I-G^{(k)}\right\|$ & & $\left\|I-G^{(k)}\right\|$ \\
\hline 1 & $1.71 \cdot 10^{91}$ & $9.22 \cdot 10^{92}$ & $1.00 \cdot 10^{00}$ & & $1.00 \cdot 10^{00}$ \\
2 & $1.22 \cdot 10^{81}$ & $1.02 \cdot 10^{83}$ & $1.00 \cdot 10^{00}$ & & $1.00 \cdot 10^{00}$ \\
3 & $1.11 \cdot 10^{71}$ & $1.14 \cdot 10^{73}$ & $1.00 \cdot 10^{00}$ & & $1.00 \cdot 10^{00}$ \\
4 & $1.10 \cdot 10^{61}$ & $1.27 \cdot 10^{63}$ & $1.00 \cdot 10^{00}$ & & $1.00 \cdot 10^{00}$ \\
5 & $1.14 \cdot 10^{51}$ & $1.41 \cdot 10^{53}$ & $1.00 \cdot 10^{00}$ & & $1.00 \cdot 10^{00}$ \\
6 & $1.21 \cdot 10^{41}$ & $1.56 \cdot 10^{43}$ & $1.00 \cdot 10^{00}$ & & $1.00 \cdot 10^{00}$ \\
7 & $1.31 \cdot 10^{31}$ & $1.74 \cdot 10^{33}$ & $1.00 \cdot 10^{00}$ & & $1.00 \cdot 10^{00}$ \\
8 & $1.43 \cdot 10^{21}$ & $1.93 \cdot 10^{23}$ & $1.00 \cdot 10^{00}$ & & $1.00 \cdot 10^{00}$ \\
9 & $1.58 \cdot 10^{11}$ & $2.15 \cdot 10^{13}$ & $1.00 \cdot 10^{00}$ & & $1.00 \cdot 10^{00}$ \\
10 & $1.84 \cdot 10^{01}$ & $2.38 \cdot 10^{03}$ & $9.46 \cdot 10^{-01}$ & & $9.46 \cdot 10^{-01}$ \\
11 & $1.00 \cdot 10^{00}$ & $2.65 \cdot 10^{-07}$ & $2.05 \cdot 10^{-09}$ & & $3.88 \cdot 10^{-16}$ \\
12 & $1.00 \cdot 10^{00}$ & $2.94 \cdot 10^{-17}$ & $1.11 \cdot 10^{-10}$ & \\
\hline
\end{tabular}

Table IV. Result for Rump matrix randmatsym ( $n$, cnd) with $n=500$, several anticipated condition numbers $c$ nd and $\varepsilon_{t o l}=10^{-10} .\left(t_{i}\right.$ : elapsed time [sec], $m_{i}:$ number of iterations.)

\begin{tabular}{|c|c|c|c|c|c|c|c|}
\hline \multirow[b]{2}{*}{ cnd } & \multirow[b]{2}{*}{$\kappa(A)$} & \multicolumn{3}{|c|}{ Algorithm 1} & \multicolumn{3}{|c|}{ Algorithm 2} \\
\hline & & $t_{1}$ & $m_{1}$ & $\left\|I-G^{\left(m_{1}\right)}\right\|$ & $t_{2}$ & $m_{2}$ & $\left\|I-G^{(M)}\right\|$ \\
\hline $10^{20}$ & $5.76 \cdot 10^{21}$ & 2.24 & 3 & $2.78 \cdot 10^{-11}$ & 1.35 & 3 & $4.44 \cdot 10^{-16}$ \\
\hline $10^{40}$ & $3.32 \cdot 10^{43}$ & 5.74 & 5 & $2.78 \cdot 10^{-11}$ & 4.28 & 5 & $4.44 \cdot 10^{-16}$ \\
\hline $10^{60}$ & $3.26 \cdot 10^{63}$ & 11.93 & 7 & $2.78 \cdot 10^{-11}$ & 9.70 & 7 & $4.44 \cdot 10^{-16}$ \\
\hline $10^{80}$ & $1.10 \cdot 10^{87}$ & 24.82 & 10 & $2.78 \cdot 10^{-11}$ & 18.06 & 9 & $4.44 \cdot 10^{-16}$ \\
\hline $10^{100}$ & $3.18 \cdot 10^{99}$ & 33.45 & 11 & $2.78 \cdot 10^{-11}$ & 27.92 & 11 & $4.44 \cdot 10^{-16}$ \\
\hline
\end{tabular}

Table V. Result for Rump matrix randmatsym $(n, c n d)$ with several $n$ and $\kappa(A) \approx 10^{19}$. ( $t_{i}$ : elapsed time [sec], $m_{i}:$ number of iterations.)

\begin{tabular}{ccccccccc}
\hline & & \multicolumn{3}{c}{ Algorithm 1} & & \multicolumn{3}{c}{ Algorithm 2} \\
\cline { 3 - 4 }$n$ & $\varepsilon_{\text {tol }}$ & $t_{1}\left(t_{0}\right)$ & $m_{1}\left(m_{0}\right)$ & $\left\|I-G^{\left(m_{1}\right)}\right\|$ & & $t_{2}$ & $m_{2}$ & $\left\|I-G^{(M)}\right\|$ \\
\hline 500 & $10^{-10}$ & $2.19(2.20)$ & $3(3)$ & $2.85 \cdot 10^{-11}$ & & 1.31 & 3 & $4.44 \cdot 10^{-16}$ \\
1000 & $10^{-9}$ & $17.41(17.00)$ & $3(3)$ & $1.57 \cdot 10^{-10}$ & & 9.12 & 3 & $6.67 \cdot 10^{-16}$ \\
2000 & $10^{-9}$ & $93.66(92.82)$ & $3(3)$ & $6.55 \cdot 10^{-10}$ & & 52.25 & 3 & $4.80 \cdot 10^{-16}$ \\
3000 & $10^{-8}$ & $247.40(239.42)$ & $3(3)$ & $1.10 \cdot 10^{-09}$ & & 145.31 & 3 & $4.80 \cdot 10^{-16}$ \\
4000 & $10^{-8}$ & $1068.87(643.89)$ & $4(3)$ & $1.78 \cdot 10^{-09}$ & & 786.92 & 4 & $4.44 \cdot 10^{-16}$ \\
5000 & $10^{-8}$ & $2301.48(1338.25)$ & $4(3)$ & $2.78 \cdot 10^{-09}$ & & 1676.50 & 4 & $9.77 \cdot 10^{-16}$ \\
\hline
\end{tabular}

condition numbers cnd from $10^{20}$ to $10^{100}$. The results are displayed in Table IV. As can be seen, $t_{1}$ and $t_{2}$ gradually increase according to the condition numbers, and $t_{2}$ is smaller than $t_{1}$. This is because Algorithm 1 needs to calculate (13) which requires $\mathcal{O}\left(m_{1}^{2} n^{3}\right)$ flops, while Algorithm 2 does not. Namely, in the case of $m_{1}=M$, the computational cost of Algorithm 2 is less than that of Algorithm 1. Second, we fix $c n d=10^{19}$ and vary $n$ from 500 to 5000. The results are displayed in Table V. As expected, $t_{2}$ is smaller than $t_{1}$, which is a similar result to the previous example.

It turns out that Algorithm 2 is faster and gives more accurate results than Algorithm 1. All the numerical results shown in Tables I - V are consistent with our analysis mentioned in Section 3.

\section{Conclusion}

We propose a modified version of the Ogita-Oishi's algorithm [2] for calculating an accurate inverse of the exact Cholesky factor for ill-conditioned matrices. The presented analysis shows that the proposed algorithm (Algorithm 2) gives more accurate results than the original one (Algorithm 1). 
The numerical results are consistent with our analysis. Moreover, the computational cost of Algorithm 2 is less than that of Algorithm 1. Thus, Algorithm 2 is faster than Algorithm 1.

One may be interested in comparing accurate inverse Cholesky factorizations with a standard Cholesky factorization applying long precision arithmetic such as GMP [16] and MPFR [17]. In [4], such numerical results for Algorithm 1 are presented in terms of measured computing time for verifying the positive definiteness of an input matrix. For Algorithm 2, we expect that similar results to those in [4] are provided.

\section{References}

[1] W.F. Alizadeh, "Interior point methods in semidefinite programming with application to combinatorial optimization," SIAM Journal on Optimization, vol. 5, pp. 13-51, 1995.

[2] T. Ogita and S. Oishi, "Accurate and robust inverse Cholesky factorization," NOLTA, vol. 3, pp. 103-111, 2012.

[3] Y. Yanagisawa and T. Ogita, "Convergence analysis of accurate inverse Cholesky factorization," JSIAM Letters, vol. 5, pp. 25-28, 2013.

[4] Y. Yanagisawa and T. Ogita, "Convergence analysis of an algorithm for accurate inverse Cholesky factorization," submitted for publication.

[5] N.J. Higham, Accuracy and Stability of Numerical Algorithms, 2nd ed., SIAM, Philadelphia, PA, 2002.

[6] S.M. Rump, "Inversion of extremely ill-conditioned matrices in floationg-point," Japan J. Indust. Appl. Math., vol. 26, pp. 249-277, 2009.

[7] T. Ogita, S.M. Rump, and S. Oishi, "Accurate sum and dot product," SIAM J. Sci. Comput., vol. 26, pp. 1955-1988, 2005.

[8] S.M. Rump, T. Ogita, and S. Oishi, "Accurate floating-point summation part I: faithful rounding," SIAM J. Sci. Comput., vol. 31, pp. 189-224, 2008.

[9] S.M. Rump, T. Ogita, and S. Oishi, "Accurate floating-point summation part II: sign, K-fold faithful and rounding to nearest," SIAM J. Sci. Comput., vol. 31, pp. 1269-1302, 2008.

[10] K. Ozaki, T. Ogita, S. Oishi, Siegfried M. Rump, "Error-free transformations of matrix multiplication by using fast routines of matrix multiplication and its applications," Numerical Algorithms, vol. 59, pp. 95-118, 2012.

[11] S.M. Rump, T. Ogita, "Super-fast validated solution of linear systems," J. Comput. Appl. Math., vol. 199, no. 2, pp. 199-206, 2007.

[12] S.M. Rump, "Verification of positive definiteness," BIT Numerical Mathematics, vol. 46, pp. 433-452, 2006.

[13] G.W. Stewart and J.-G. Sun, Matrix Perturbation Theory, Academic Press, Boston, 1990.

[14] S.M. Rump, "A class of arbitrarily ill-conditioned floating-point matrices," SIAM J. Matrix Anal. Appl., vol. 12, no. 4, pp. 645-653, 1991.

[15] S.M. Rump, "INTLAB - INTerval LABoratory," Developments in Reliable Computing, ed. T. Csendes, Kluwer Academic Publishers, Dordrecht, pp. 77-104, 1999.

[16] GMP, GNU Multiple Precision Arithmetic Library, version 5.1.0, 2012. Code and documentation available at http://gmplib.org/.

[17] MPFR, The Multiple Precision Floating-Point Reliable Library, version 3.1.2, March 2013. Code and documentation available at http://www.mpfr.org/. 